\title{
Geotechnical reliability updating using stochastic FEM
}

\author{
I. Papaioannou \\ Computation in Engineering, TU München, Germany \\ D. Straub \\ Engineering Risk Analysis Group, TU München, Germany
}

\begin{abstract}
In geotechnical sites, deformation measurements are commonly made during the construction process. In this paper, it is shown how information obtained during such measurements can be utilized to update the estimate of the reliability at future construction stages. A recently proposed method for Bayesian updating of the reliability with information of equality type is successfully applied in conjunction with a stochastic nonlinear geotechnical finite element model. Therein uncertainty in the soil material properties is modelled by non-Gaussian random fields. The conditional reliability is evaluated by means of an efficient adaptive Monte Carlo method, known as subset simulation. The approach is demonstrated through an application to a sheet pile wall at a deformation-sensitive geotechnical construction site.
\end{abstract}

\section{INTRODUCTION}

Geotechnical engineers often use the observation method to deal with uncertainties in site conditions or performance behavior. The engineer collects a few hypotheses about site conditions and then gathers field observations to identify the correct hypothesis. In probability theory, Bayesian updating formalizes this procedure, by quantifying the effect of an observation on the probability of a prior hypothesis. Bayesian updating is applied here within the structural reliability concept. To this end, a recently proposed methodology (Straub 2010) that allows updating of the reliability given measurement information is applied. Hence, the effect of measurements at a geotechnical construction site on the failure probability of the system is quantified.

In geotechnical reliability analysis, material nonlinearities are often taken into account, leading to failure events defined by nonlinear performance functions. The largest uncertainties in the analysis stem from the mechanical properties of the soil materials. The inherent spatial variability of the soil necessitates the consideration of random fields for the accurate modeling of these uncertainties. Moreover, the consideration of the spatial variability seems to have an important influence on the computed reliability (Rackwitz 2000).

In the context of finite element $(\mathrm{FE})$ reliability analysis, the stochastic FE discretization of the random fields may lead to a large number of random variables. The reliability of such highdimensional nonlinear problems can be solved efficiently by either first or second order reliability methods combined with the direct differentiation method for the evaluation of the derivatives (Zhang \& Der Kiureghian 1993, Der Kiureghian \& Zhang 1999) or by certain simulation approaches, such as the subset simulation, the spherical subset simulation and recently the asymptotic sampling method (Au \& Beck 2001, Katafygiotis \& Cheung 2007, Bucher 2009). The former case requires alterations at the FE code level, while the latter may be easily coupled with a "black-box" FE code.

In this work, the subset simulation is applied for the reliability updating of a geotechnical FE model, involving an excavation in sand with a sheet pile retaining wall, whereby the underlying non-Gaussian random fields are discretized using the stochastic FE method. 


\section{STOCHASTIC FEM}

In computational mechanics, the continuous nature is approximated by a discrete representation through the discretization procedure. Among the different discretization methods, the FE method is considered the most suitable for application in geotechnical engineering (Zienkiewicz \& Taylor 2000). The FE method requires the splitting of the continuous medium into an assembly of individual elements, i.e. the FE mesh. Moreover, nonlinear FE analysis computes the deformations and stresses through the solution of a nonlinear system of equations, wherein the nonlinear effects of the soil material properties as well as the interaction between the soil and adjacent retaining structures are taken into account. For the simulation of construction sites, usually a sequence of nonlinear FE analyses is performed to model the different stages of the construction process.

The consideration of the stochastic nature of the mechanical properties of soil requires an additional discretization of the relevant random fields. A random field $X(\mathbf{t})$ may be loosely defined as a random function, which describes a random variable at each point $\mathbf{t}$ of a continuous space, where $\mathbf{t}$ is a location vector. To completely define a random field, the joint distribution of the random variables $\left\{X\left(\mathbf{t}_{1}\right), X\left(\mathbf{t}_{2}\right) \ldots, X\left(\mathbf{t}_{n}\right)\right\}$ for any $\left\{n, \mathbf{t}_{1}, \mathbf{t}_{2}, \ldots, \mathbf{t}_{n}\right\}$ must be specified. In most engineering applications, however, the joint distribution is modeled by a Gaussian copula (the Nataf distribution, Der Kiureghian \& Liu 1986), in which case the distribution is completely described by second moment information and the marginal distribution. Denoting the expectation operator by E[.], we define the mean and auto-covariance function of the random field respectively by:

$$
\begin{aligned}
& \mu_{X}(\mathbf{t})=\mathrm{E}[X(\mathbf{t})] \\
& \left.\gamma_{X X}(\mathbf{t}, \mathbf{s})=\mathrm{E}[\{X(\mathbf{t})-\mu(\mathbf{t})\}, X(\mathbf{s})-\mu(\mathbf{s})\}\right]
\end{aligned}
$$

The variance function is expressed as:

$$
\sigma_{X}^{2}(\mathbf{t})=\gamma_{X X}(\mathbf{t}, \mathbf{t})
$$

The auto-correlation coefficient function may then be defined by:

$$
\rho_{X X}(\mathbf{t}, \mathbf{s})=\frac{\gamma_{X X}(\mathbf{t}, \mathbf{s})}{\sigma_{X}(\mathbf{t}) \sigma_{X}(\mathbf{s})}
$$

A random field is said to be second-order homogeneous if its second-order properties are invariant to a shift of the origin. A direct consequence of this is that the mean and variance are constant over space and the auto-covariance and auto-correlation coefficient functions depend only on the difference in location $\tau$ between two points:

$$
\begin{aligned}
& \left.\gamma_{X X}(\boldsymbol{\tau})=\mathrm{E}\left[\left\{X(\mathbf{t}+\boldsymbol{\tau})-\mu_{X}\right\} X X(\mathbf{t})-\mu_{X}\right\}\right] \\
& \rho_{X X}(\boldsymbol{\tau})=\frac{\gamma_{X X}(\boldsymbol{\tau})}{\sigma_{X}^{2}}
\end{aligned}
$$

The continuous random field $X(\mathbf{t})$ may be approximated by a discrete $\hat{X}(\mathbf{t})$, defined by means of a finite set of random variables $X_{i}$. Several methods have been proposed for the discretization of random fields - a comprehensive review is given by Sudret \& Der Kiureghian (2000). In this study, the midpoint method (Der Kiureghian \& Ke 1988) is adopted. This method requires a stochastic finite element (SFE) mesh, which can be independent of the FE mesh used in the deterministic part of the analysis. According to this method, the value of the random field $X(\mathbf{t})$ over each SFE $i$ is represented by its value at the midpoint $\mathbf{t}_{i}$ of the element:

$$
X_{i}=X\left(\mathbf{t}_{i}\right)
$$

If the random field is second-order homogeneous, the mean and the variance of the random variables $X_{i}$ are not affected by the discretization and therefore are equal to their constant values over the entire field. The correlation matrix of the random variables is generated by computing the distances $\boldsymbol{\tau}_{i j}$ between the midpoints of each element and inserting into Equation 6. The ran- 
dom field is thus reduced to a vector $\mathbf{X}$ of correlated random variables with known marginal distributions and correlation coefficients.

\section{RELIABILITY ANALYSIS}

Let $\mathbf{X}$ be a vector of random variables with joint probability density function (PDF) $f_{\mathbf{X}}(\mathbf{x})$. A failure event $F$ is defined by a domain $\Omega_{F}=\{g(\mathbf{x}) \leq 0\}$ in the outcome space of $\mathbf{X}$, with $g(\mathbf{x})$ being a performance function with the following general form:

$$
g(\mathbf{x})=\min _{1 \leq k \leq K}\left[\max _{i \in C_{1}} g_{i}(\mathbf{x}) \ldots, \max _{i \in C_{K}} g_{i}(\mathbf{x})\right]
$$

where $\left\{g_{i}(\mathbf{x}), i=1, \ldots, m\right\}$ is a set of component performance functions and $C_{k}$ is an index set denoting the $k$-th cut set. The component-reliability problem may be derived by setting $i=1$, the parallel-system reliability problem by setting $K=1$, and the series-system reliability problem by defining each cut set by a single index. The probability of failure for the general reliability problem is evaluated by:

$$
\operatorname{Pr}(F)=\operatorname{Pr}\{g(\mathbf{X}) \leq 0\}=\int_{\Omega_{F}} f_{\mathbf{X}}(\mathbf{x}) \mathrm{d} \mathbf{x}
$$

In the case where only second moments and marginal distributions are available, the joint PDF may be approximated using the Nataf model and a transformation of the random variables from the original random variable space to an equivalent independent standard Gaussian space (transformed space) $\mathbf{U}=T(\mathbf{X})$ may be performed (Der Kiureghian \& Liu 1986). The probability may then be computed as follows:

$$
\operatorname{Pr}(F)=\int_{G(\mathbf{u}) \leq 0} \varphi_{\mathbf{U}}(\mathbf{u}) \mathrm{d} \mathbf{u}
$$

where $\varphi_{\mathbf{U}}(\mathbf{u})$ is the standard normal joint PDF and $G(\mathbf{u})=g\left[T^{-1}(\mathbf{u})\right]$.

\subsection{Reliability updating with equality information}

Consider measurements or other observations of the structural or geotechnical system. This information is described by an event $Z$, which in structural reliability is defined through a domain $\Omega_{Z}$ in the outcome space of the basic random variables $\mathbf{X}$. The conditional probability of $F$ given the information $Z$ is:

$$
\operatorname{Pr}(F \mid Z)=\frac{\operatorname{Pr}(F \cap Z)}{\operatorname{Pr}(Z)}=\frac{\int_{\Omega_{F} \cap \Omega_{Z}} f_{\mathbf{X}}(\mathbf{x}) \mathrm{d} \mathbf{x}}{\int_{\Omega_{Z}} f_{\mathbf{X}}(\mathbf{x}) \mathrm{d} \mathbf{x}}
$$

The domain $\Omega_{Z}$ is defined through a function $h(\mathbf{x})$; if $\Omega_{Z}$ is defined as $\Omega_{Z}=\{h(\mathbf{x}) \leq 0\}$, the event $Z$ is said to be of inequality-type and if $\Omega_{Z}$ is defined as $\Omega_{Z}=\{h(\mathbf{x})=0\}, Z$ is said to be of equality-type. In the latter case, the probability of the event $Z$ is zero and both integrals in Equation 11 will result in zero. To circumvent this problem, a method proposed in (Straub 2010) will be applied, according to which the event $Z$ is replaced by an equivalent inequality-type event $Z_{e}$.

To illustrate the methodology, consider the case where $Z$ describes the measurement $s_{m}$ of a system characteristic $s(\mathbf{x})$, e.g. a deformation measurement of a structure, with corresponding equality-type performance function:

$$
h\left(\mathbf{x}, \varepsilon_{m}\right)=s(\mathbf{x})-s_{m}+\varepsilon_{m}
$$

where $\varepsilon_{m}$ is a random variable representing the measurement error. Equivalently, the information of the event $Z$ with respect to the random variables $\mathbf{X}$ can be expressed by the likelihood function:

$$
L(\mathbf{x})=f_{\varepsilon_{m}}\left[s_{m}-s(\mathbf{x})\right]
$$


where $f_{\varepsilon m}$ is the PDF of $\varepsilon_{m}$. It is suggested in (Straub 2010) to utilize this likelihood function to define a new performance function:

$$
h_{e}(\mathbf{x}, u)=u-\Phi^{-1}[c L(\mathbf{x})]
$$

where $u$ is the outcome of a standard normal random variable, $\Phi^{-1}($.$) is the inverse of the stan-$ dard normal cumulative distribution function and $c$ is a positive constant, chosen to ensure that $c L(\mathbf{x}) \leq 1$ for all $\mathbf{x}$. Let $\Omega_{Z e}=\left\{h_{e}\left(\mathbf{x}_{+}\right) \leq 0\right\}$, be the corresponding domain of event $Z_{e}$ that defines an inequality information, where $\mathbf{x}_{+}$is the outcome of $\mathbf{X}_{+}=[\mathbf{X}, U]^{T}$. It is shown in (Straub 2010) that the conditional probability of $F$ given $Z$ is:

$$
\operatorname{Pr}(F \mid Z)=\operatorname{Pr}\left(F \mid Z_{e}\right)=\frac{\operatorname{Pr}\left(F \cap Z_{e}\right)}{\operatorname{Pr}\left(Z_{e}\right)}=\frac{\int_{\Omega_{F} \cap \Omega_{z_{e}}} f_{\mathbf{X}_{+}}\left(\mathbf{x}_{+}\right) \mathrm{d} \mathbf{x}_{+}}{\int_{\Omega_{Z_{e}}} f_{\mathbf{X}_{+}}\left(\mathbf{x}_{+}\right) \mathrm{d} \mathbf{x}_{+}}
$$

where $f_{\mathbf{X}_{+}}\left(\mathbf{x}_{+}\right)$is the joint PDF of $\mathbf{X}_{+}$. As opposed to the original formulation in Equation 11, both integrals in Equation 15 have finite value and can thus be computed using any structural reliability method, including simulation methods. Here, this formulation will be applied in conjunction with subset simulation for the application to geotechnical reliability updating.

\section{SUBSET SIMULATION}

The subset simulation is an adaptive simulation method developed by Au \& Beck (2001), which is shown to be efficient especially in high dimensions. The method is based on the standard Monte Carlo simulation (MCS) but overcomes its inefficiency in estimating small probabilities, while maintaining its independency on the problem dimensionality. This is achieved by expressing the failure event $F$ as the intersection of $M$ intermediate failure events:

$$
F=\bigcap_{i=1}^{M} F_{i}
$$

where $F_{1} \supset F_{2} \supset \cdots \supset F_{M}=F$. The probability of failure is estimated by applying the definition of conditional probability, resulting in:

$$
\operatorname{Pr}(F)=\operatorname{Pr}\left(F_{1}\right) \prod_{i=2}^{M} \operatorname{Pr}\left(F_{i} \mid F_{i-1}\right)
$$

The failure event is defined in the equivalent standard normal space by $G(\mathbf{u}) \leq 0$; each of the intermediate events is defined as $F_{i}=\left\{G(\mathbf{u}) \leq G_{i}\right\}$, where $G_{i}>\ldots>G_{M}=0$. The values of $G_{i}$ are chosen adaptively so that the estimates of the conditional probabilities correspond to a chosen value $p_{0}$. The probability of $F_{1}$ is computed by applying the crude MCS method (Rubinstein 1981). Through MCS, we simulate $N$ samples of $\mathbf{U}$ and take as $G_{1}$ the $\left[\left(1-p_{0}\right) N\right]$-th largest value among the samples $\left\{G\left(\mathbf{u}_{k}\right): k=1, \ldots, N\right\}$. The samples $\mathbf{u}_{i}$ for which $G\left(\mathbf{u}_{i}\right) \leq G_{1}$ are used as starting points for the simulation of samples conditional on $F_{1}$, by applying the MetropolisHastings algorithm, which is a Markov chain Monte Carlo technique. This procedure is repeated until the maximum level $M$ is reached, for which the threshold $G_{M}=0$ is given. The failure probability is then approximated by:

$$
\operatorname{Pr}(F) \approx p_{0}{ }^{M-1} \operatorname{Pr}\left(F_{M} \mid F_{M-1}\right)
$$

where the conditional probability $\operatorname{Pr}\left(F_{M} \mid F_{M-1}\right)$ is equal to the ratio of the number of samples for which $G(\mathbf{u}) \leq 0$ over the total number of samples $N$ simulated conditional on $F_{M-1}$.

The choice of $p_{0}$ and the number of samples at each step $N$ may in principle be arbitrary. However, the choice of $N$ should be large enough to give an accurate estimation of $p_{0}$. In this study, the values $p_{0}=0.1$ and $N=500$ were chosen, following the initial suggestions given by Au \& Beck (2001). 


\section{APPLICATION}

The application considered is a deep excavation in sand with a sheet pile retaining wall. The FE model was built in the SOFiSTiK program and the reliability assessment was performed using a reliability tool, developed as part of the SOFiSTiK software package (Papaioannou et al. 2009).

\subsection{Geotechnical model description}

The site consists of a $5.0 \mathrm{~m}$ deep trench with cantilever sheet piles, without anchors or bottom support (Fig. 1), in a homogeneous soil layer of dense cohesionless sand with uncertain spatially varying mechanical properties. The soil is modeled in $2 \mathrm{D}$ with plane-strain finite elements. For simplicity, neither groundwater nor external loading is considered. The material model used is an elastoplastic model with a prismatic yield surface according to the Mohr-Coulomb criterion and a non-associated plastic flow. The probability distributions, describing the uncertainty in the material properties of the soil, are shown in Table 1 . The spatial variability of the soil is modeled by a second-order homogeneous random field, with the following exponential autocorrelation coefficient function (Vanmarcke 1983):

$$
\rho_{X X}(\boldsymbol{\tau})=\exp \left(-\frac{\tau_{x}}{l_{x}}-\frac{\tau_{z}}{l_{z}}\right)
$$

where $\tau=\left[\tau_{x}, \tau_{z}\right]^{T}$ is the vector of absolute distances in the $x$ (horizontal) and $z$ (vertical) directions. The correlation lengths were chosen as $l_{x}=20 \mathrm{~m}$ and $l_{z}=5 \mathrm{~m}$. The random field was discretized by the midpoint method using a SFE mesh, consisting of 144 deterministic FE patches. The stochastic discretization resulted in a total of 432 basic random variables. In Figure 2, the stochastic and deterministic FE meshes are shown.

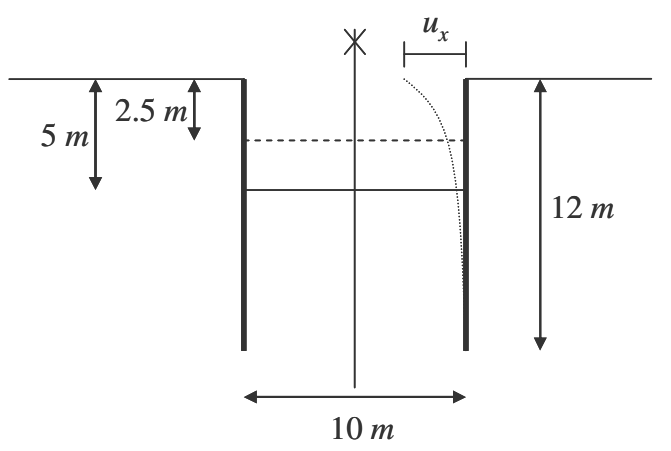

Dense cohesionless sand

Figure 1. Sheet pile wall in sand.

Table 1. Material properties.

\begin{tabular}{llll}
\hline Parameter & Distribution & Mean & COV \\
\hline Specific weight $\gamma\left[\mathrm{kN} / \mathrm{m}^{3}\right]$ & Normal & 19.0 & $5 \%$ \\
Young's modulus $E[\mathrm{MPa}]$ & Lognormal & 125.0 & $25 \%$ \\
Poisson's ratio $v$ & - & 0.35 & - \\
Friction angle $\varphi\left[{ }^{\circ}\right]$ & Beta $(0.0,45.0)$ & 35.0 & $10 \%$ \\
Cohesion $c[\mathrm{MPa}]$ & - & 0.0 & - \\
Dilatancy angle $\psi\left[{ }^{\circ}\right]$ & - & 5.0 & - \\
\hline
\end{tabular}

A deterministic design using the conventional method for cantilever sheet pile design in granular soils, which requires equilibrium of the active and passive lateral pressures (e.g. see Tschebotarioff 1951), was performed analytically. A depth of $7.5 \mathrm{~m}$ and the sheet pile profile PZC 13 were chosen, applying a global safety factor of 1.5. The Young's modulus of steel was taken as $210 \mathrm{GPa}$. The pile was modeled using beam elements with an equivalent rectangular cross sec- 
tion that behaves equally to the sheet pile in bending and axial resistance. The interaction between the retaining structure and the surrounding soil was modeled using nonlinear interface elements. An elastoplastic model with a yield surface defined by the Mohr-Coulomb criterion is used to describe the interface behavior. The elastic properties of the interface elements are taken from the mean values of the adjacent soil, while the strength properties are reduced by the factor $2 / 3$ and a zero dilatancy is chosen.

The finite element analysis is performed stepwise. First, the modeling of the in-situ stress state is carried out applying the $K_{0}$-procedure, where $K_{0}$ is the lateral earth pressure coefficient at rest, computed here using the expression proposed by Jaky (1944) for normally consolidated soils:

$$
K_{0}=1-\sin \varphi
$$

Next, the sheet pile is installed by activating the corresponding beam and interface elements. Finally, the excavation is modeled by removing the plane-strain elements corresponding to the trench and applying the necessary loading to establish equilibrium.

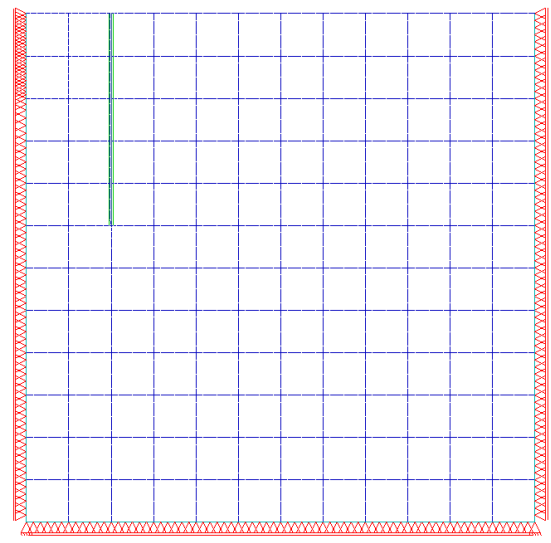

(a) Stochastic finite element mesh

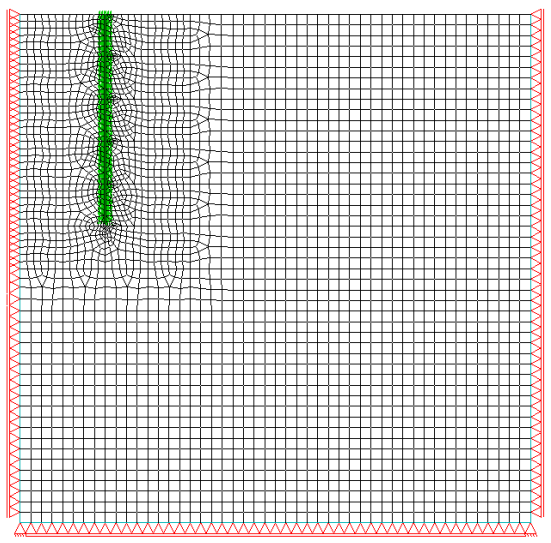

(b) Deterministic finite element mesh

Figure 2. Stochastic and deterministic finite element mesh.

\subsection{Limit state functions}

The maximum horizontal $u_{x}$ displacement occurs at the top of the trench. The failure event $F$ is defined as the event of $u_{x}$ exceeding a threshold of $u_{x, t}=10 \mathrm{~cm}$. Mathematically, this is expressed through the following performance function:

$$
g(\mathbf{x})=u_{x, t}-u_{x}(\mathbf{x})
$$

We assume that a measurement of the displacement $u_{x}$ is made at an intermediate excavation step of $2.5 \mathrm{~m}$ depth. This information is expressed by an event $Z$, defined through the following equality-type performance function:

$$
h(\mathbf{x})=u_{x}(\mathbf{x})-u_{x, m}+\varepsilon_{m}
$$

where $\varepsilon_{m}$ is a zero mean Gaussian random variable, representing the measurement error. The corresponding equivalent inequality performance function is:

$$
h_{e}(\mathbf{x}, u)=u-\Phi^{-1}\left[\frac{c}{\sigma_{\varepsilon, m}} \varphi\left(\frac{u_{x, m}-u_{x}(\mathbf{x})}{\sigma_{\varepsilon, m}}\right)\right]
$$

where $\sigma_{\varepsilon, m}$ is the standard deviation of $\varepsilon_{m}$ and the constant is chosen as $c=\sigma_{\varepsilon, m}$. 


\subsection{Results}

In Figure 3, the deformed configuration is depicted at the final excavation stage for the mean values of the random fields.

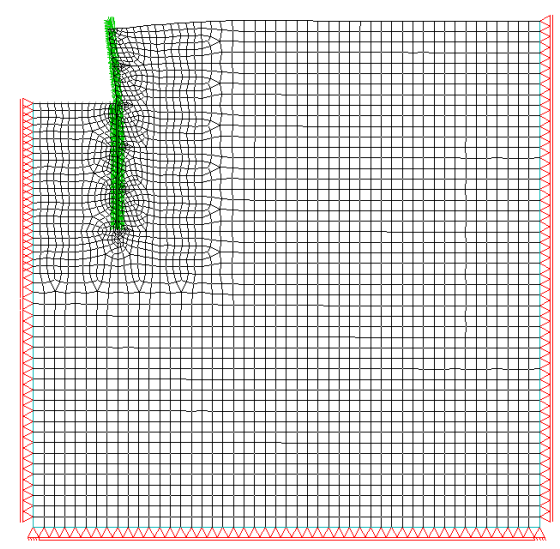

Figure 3. Deformed configuration at the final excavation step.

The reliability analysis is performed by means of subset simulation. Without measurements, the computed failure probability is $\operatorname{Pr}(F)=1.360 \times 10^{-2}$ with a corresponding reliability index $\beta=$ 2.209 .

For the estimation of the updated failure probability conditional on the measurement event $Z$, the integrals in Equation 15 were evaluated with subset simulation. The reliability updating was performed for different values of the assumed measurement $u_{x, m}$, and different values of the standard deviation $\sigma_{\varepsilon, m}$ of the measurement error. The results are summarized in Table 2. For comparison, the (a-priori) expected value of $h(\mathbf{x})$ is computed as $2.57 \mathrm{~mm}$.

Table 2. Updated failure probability $\operatorname{Pr}(F)=1.360 \times 10^{-2}$

\begin{tabular}{lll}
\hline Measurement & $\operatorname{Pr}(F \mid Z)$ & \\
\hline & $\sigma_{\varepsilon, m}=2 \mathrm{~mm}$ & $\sigma_{\varepsilon, m}=1 \mathrm{~mm}$ \\
\cline { 2 - 3 }$u_{x, m}=10 \mathrm{~mm}$ & $2.185 \times 10^{-1}$ & $3.313 \times 10^{-1}$ \\
$u_{x, m}=5 \mathrm{~mm}$ & $2.091 \times 10^{-2}$ & $3.587 \times 10^{-2}$ \\
$u_{x, m}=2 \mathrm{~mm}$ & $6.743 \times 10^{-3}$ & $1.845 \times 10^{-3}$ \\
\hline
\end{tabular}

Not surprisingly, for measurements higher than the expected value, the updated failure probability is higher than the prior probability. This difference increases with decreasing $\sigma_{\varepsilon, m}$, representing an increase in accuracy of the measurement device. On the other hand, the updated failure probability conditional on a measurement smaller than the expected value is lower than the prior probability, and a decreasing value of $\sigma_{\varepsilon, m}$ increases this difference. It is noted, however, that a measurement that corresponds exactly to the expected value of the deformation would lead to a posterior failure probability that is lower than the prior probability, due to the reduction in uncertainty.

The number of deterministic FE analyses required by the subset simulation ranges between 1900 and 3700. The higher amount of computations is observed in the case where the assumed measurement differs considerably from the expected value (i.e. the case where $u_{x, m}=10 \mathrm{~mm}$ ). This is due to the small value of the probability $\operatorname{Pr}\left(Z_{e}\right)$ in Equation 15, resulting in a larger number of levels in the corresponding run of the subset simulation algorithm.

\section{CONCLUSION}

In this paper, a procedure for the reliability updating of geotechnical sites using measurement information is presented. The applied methodology includes the stochastic discretization of the non-Gaussian random fields, representing the spatial variability of the uncertain material pa- 
rameters. For the reliability updating, a recently proposed method that tackles Bayesian updating with equality information was applied. This approach was combined with the subset simulation, an adaptive Monte Carlo method that is able to handle efficiently reliability problems with a large number of random variables.

The proposed procedure was applied to a nonlinear stochastic finite element model of an excavation with a sheet pile retaining wall. Several different cases of deformation measurements in an intermediate construction stage were considered. The results showed that reasonable estimates of the conditional failure probability may be obtained with a feasible computational cost even when including a large number of basic random variables for the random field representation of the soil properties.

\section{ACKNOWLEDGEMENT}

This work is partially supported by SOFiSTiK AG. This support is gratefully acknowledged.

\section{REFERENCES}

Au, S.K. \& Beck, J.L. 2001. Estimation of small failure probabilities in high dimensions by subset simulation. Probabilistic Engineering Mechanics 16(4): 263-277.

Bucher, C. 2009. Asymptotic sampling for high-dimensional reliability analysis. Probabilistic Engineering Mechanics 24: 504-510.

Der Kiureghian, A. \& Ke, J-B. 1988. The stochastic finite element method in structural reliability. Probabilistic Engineering Mechanics 3(2): 83-91.

Der Kiureghian, A. \& Liu, P-L. 1986. Structural reliability under incomplete probability information. Journal of Engineering Mechanics ASCE 112(1): 85-104.

Der Kiureghian, A. \& Zhang, Y. 1999. Space variant finite element reliability analysis. Comput. Methods Appl. Mech. Engrg. 168: 173-183.

Jaky, J. 1948. The coefficient of earth pressure at rest. In Hungarian. J. Soc. Hung. Eng. Arch., 355-358.

Katafygiotis, L.S. \& Cheung, S.H. 2007. Application of spherical subset simulation method and auxiliary domain method on a benchmark reliability study. Structural Safety 29: 194-207.

Papaioannou, I., Heidkamp, H., Düster, A., Rank, E. \& Katz, C. 2009. Integration of reliability methods into a commercial finite element software package. In H. Furuta, D.M. Frangopol, M. Shonozuka (eds.), Proceedings of the $10^{\text {th }}$ International Conference on Structural Safety and Reliability ICOSSAR 2009, Osaka, September, 2009. London: Taylor \& Francis Group.

Rackwitz, R. 2000. Reviewing probabilistic soils modelling. Computers and Geotechnics 26: 199-223.

Rubinstein, R.Y. 1981. Simulation and the Monte Carlo Method. New York: John Wiley \& Sons.

Straub, D. 2010. Reliability updating with equality information, Probabilistic Engineering Mechanics, under review.

Sudret, B. \& Der Kiureghian, A. 2000. Stochastic Finite Elements and Reliability: A State-of-the-Art Report. University of California, Berkeley, 2000 - Technical Report no UCB/SEMM-2000/08.

Tschebotarioff, G.P. 1951. Soil Mechanics, Foundations and Earth Structures. New York: McGraw-Hill. Vanmarcke, E.H. 1983. Random Fields: Analysis and Synthesis. Cambridge, MA: MIT Press.

Zhang, Y. \& Der Kiureghian A. 1993. Dynamic response sensitivities of inelastic structures. Comput. Methods Appl. Mech. Engrg. 108: 23-36.

Zienkiewicz, O.C. \& Taylor, R.L. 2000. The Finite Element Method. Fifth edition. Oxford: ButterworthHeinemann. 